\title{
APLICAÇÃO DA METODOLOGIA GDE EM UMA EDIFICAÇÃO PÚBLICA- ESTUDO DE CASO: CURVELO MG
}

\author{
Estudante de Graduação \\ CEFET/MG \\ Minas Gerais; Brasil \\ silvia.gpacheco@hotmail.com \\ Professor EBTT \\ CEFET/MG \\ Minas Gerais; Brasil \\ marcos.ramos@cefetmg.br \\ MARTINI, RACHEL \\ Professora EBTT \\ CEFET/MG \\ Minas Gerais; Brasil \\ martini@cefetmg.br
}

GREGÓRIO PACHECO, SILVIA

DE PAULO RAMOS, MARCOS

\author{
GONÇALVES FILHO, EDSON \\ Estudante de Graduação \\ CEFET/MG \\ Minas Gerais; Brasil \\ edson.gonfilho@gmail.com \\ PENA BORTONE, THIAGO \\ Professor EBTT \\ CEFET/MG \\ Minas Gerais; Brasil \\ thiago.bortone@cefetmg.br \\ FERREIRA, LUCIANA
Professora EBTT
CEFET-MG - Curvelo
Minas Gerais; Brasil
lupiferreira@ cefetmg.br
}

\section{RESUMO}

A concepção de uma edificação compõe-se das fases de projeto, especificação de materiais, execução e manutenção. Caso ocorram falhas, em uma ou mais destas fases, podem surgir patologias que comprometem a segurança, a durabilidade e o desempenho futuro da edificação. $O$ presente trabalho tem como objetivo analisar a condição estrutural de um edifício em concreto armado, utilizando a Metodologia GDE/UnB (Grau de Deterioração do Elemento). Para o seu desenvolvimento, na primeira etapa, foram detalhadas, através de pesquisas bibliográficas, as características dessa metodologia e as principais causas das manifestações patológicas que podem ocorrer nesse tipo de estrutura. $\mathrm{Na}$ segunda etapa, realizou-se a inspeção predial do Prédio Administrativo do CEFET/MG - Campus Curvelo, de forma a identificar as anomalias e as falhas. Na terceira etapa, fez-se a avaliação da condição estrutural desse edifício e a elaboração do laudo de inspeção. Na última etapa determinou o grau de deterioração da estrutura com a medida a ser tomada no edifício em questão. Este trabalho auxilia no cadastramento das patologias desta edificação, assim como no acompanhamento de suas evoluções, além de ser uma importante ferramenta para o processo de reabilitação.

Palavras-chave: inspeção predial, condição estrutural, concreto armado.

\begin{abstract}
The design of a building is made up of the project design, materials specification, execution and maintenance. If failures occur in one or more of these phases may arise pathologies that compromise safety, durability and the future performance of the building. The present work aims to analyze the structural condition of a reinforced concrete building using the GDE (Element Deterioration Degree) Methodology / UnB. For its development, in the first stage, through bibliographical research, the characteristics of this methodology and the main causes of the pathological manifestations that may occur in this type of structure were detailed. In the second stage, the building was inspected to identify anomalies and faults. In the third stage, the structural condition of this building was evaluated, and the inspection report was prepared. In the last step it determined the degree of deterioration of the structure with the measure to be taken in the building in question. This work assists in the registration of the pathologies of this building, as well as the monitoring of their evolution, as well as being an important tool for the rehabilitation process.
\end{abstract}




\section{INTRODUÇÃO}

O concreto já foi conhecido como um material livre de falhas e anomalias, que não sofria deterioração. Este pensamento foi criado a partir de algumas observações de suas características, como a durabilidade e resistência. Em antigos métodos ocorriam exageros nos cálculos, o que gerava peças estruturais bem robustas, resultado de cálculos com margens muito altas de segurança (HELENE, 1992).

Quando dimensionadas, executadas e utilizadas de forma correta as estruturas de concreto armado devem resistir às ações mecânicas e à agressividade ambiental. Os fatores como falta de conhecimento adequado dos processos de construção, do comportamento dos materiais utilizados e negligências quanto à utilização das normas técnicas, são os principais motivos que provocam a diminuição da vida útil da estrutura. É comum que proprietários de imóveis, usuários e gestores prediais negligenciem atividades preventivas, corretivas, dentre outras, que poderiam melhorar o desempenho nos elementos construtivos (IBAPE, 2012).

Com o intuito de aumentar a vida útil da edificação, de acordo com a NBR 5674 (ABNT, 2012), tem-se necessidade de realizar manutenções nas edificações, uma vez que a manutenção visa preservar ou recuperar as condições de uso previsto para as edificações, incluindo todos os serviços realizados para prevenir ou corrigir a perda de desempenho decorrente da deterioração dos seus componentes, ou de atualizações nas necessidades dos seus usuários.

Uma metodologia utilizada para realizar a inspeção predial é a metodologia criada no Programa de Pós-Graduação em Estruturas e Construção Civil da Universidade de Brasília (PECC-UnB) com o intuito de realizar a avaliação quantitativa do grau de deterioração de estruturas de concreto, denominada metodologia de Grau de Deterioração do Elemento (GDE/UnB) (CASTRO, 1994).

O presente trabalho tem como objetivo analisar a condição estrutural de um edifício em concreto armado, utilizando a Metodologia GDE/UnB (Grau de Deterioração do Elemento), de forma a auxiliar no cadastramento das patologias desta edificação, assim como no acompanhamento de suas evoluções. Além de ser uma importante ferramenta para o processo de reabilitação.

\section{METODOLOGIA DE INSPEÇÃO EM EDIFICAÇÕES GDE/UnB}

O Grau de Deterioração do Elemento (GDE/UnB) é uma metodologia desenvolvida por Castro (1994) com o intuito de quantificar o grau de deteroriação da estrutura como um todo. Para tal, a estrutura é subdivida em subgrupos chamados de elementos e, de acordo com o tipo de elemento se formam as famílias de elementos.

Essa metodologia é de aplicação visual onde são atribuídos fatores de ponderação $\left(\mathrm{F}_{\mathrm{p}}\right)$ e fatores de intensidade $\left(\mathrm{F}_{\mathrm{i}}\right)$ para cada uma das patologias identificadas na estrutura, a fim de se calcular o grau de Dano (Equação 1). Após calculado o grau de Dano (D), o próximo passo é o cálculo do grau de deteorioração do elemento (Equação 2) e da família de elementos (Equação 3) para, por fim, calcular o grau de deterioração da estrutura (Equação 4), conforme o fluxograma da Figura 1. 


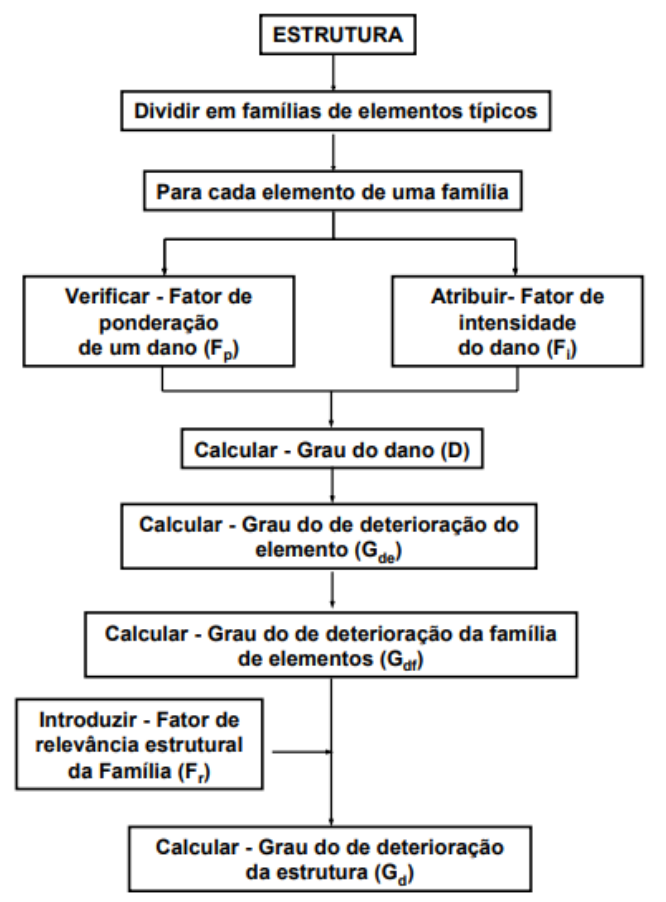

Figura 1- Fluxograma da metodologia para o cálculo do Grau de Deterioração da Estrutura (Gd).

Fonte: CASTRO (1994).

\subsection{Fator de Intensidade e ponderação do dano}

De acordo com as consequências das patologias em relação à condição estética a metodologia estabelece valores para o fator de ponderação $\left(\mathrm{F}_{\mathrm{p}}\right)$, que podem variar de 1 a 5 , já o fator de intensidade $\left(\mathrm{F}_{\mathrm{i}}\right)$ determina as possíveis lesões que o elemento pode apresentar e varia de 0 a 4 , conforme a Tabela 1 :

Tabela 1 - Classificação do Fator de Intensidade $\left(\mathrm{F}_{\mathrm{i}}\right)$.

\begin{tabular}{c|c}
\hline $\mathrm{F}_{\mathrm{i}}$ & Lesões \\
\hline 0 & Sem lesões \\
\hline 1 & Lesões leves \\
\hline 2 & Lesões toleráveis \\
\hline 3 & Lesões graves \\
\hline 4 & Estado Crítico \\
\hline & Fonte: CASTRO (1994).
\end{tabular}

Essa metodologia sofreu modificações por Lopes (1998), quando observado que este critério poderia levar, algumas vezes a resultados incoerentes. Dessa maneira, Lopes (1998) modificou a fórmula do grau de deterioração do elemento $\left(\mathrm{G}_{\mathrm{de}}\right)$. Boldo (2002), utilizou o princípio de Lopes (1998) para o grau de deterioração da família $\left(\mathrm{G}_{\mathrm{df}}\right)$, em diferentes edificações, alterando-se sua denominação no Caderno de Inspeções para Inspeção para Estruturas de Concreto. Posteriormente, Fonseca (2007) utilizou a metodologia GDE/UnB para calcular o grau de deterioração de estruturas de concreto armado, além de alterar as formulações dos cálculos do Grau de deterioração da família $\left(G_{\text {df }}\right)$ e do Grau do Dano (D) e apresentar uma mudança nos tipos mais usuais de danos. (BOAS et al., 2018). Para a avaliação de estruturas em Concreto Armado, foram utilizadas as seguintes formulações, adaptadas por Fonseca (2007):

\subsection{Grau de Dano (D)}

$$
D=0,8 F_{i} F_{p} \quad \text { para } F_{i} \leq 2
$$




$$
D=\left(12 F_{i}-28\right) F_{p} \quad \text { para } \mathrm{F}_{\mathrm{i}}>2
$$

Sendo:

D: Grau do Dano;

$F_{\mathrm{i}}$ : Fator de Intensidade;

$\mathrm{F}_{\mathrm{p}}$ : Fator de Ponderação.

\subsection{Grau de Deterioração de um elemento $\left(G_{d e}\right)$}

$$
G_{d e}=D_{\max }\left[1+\frac{\sum_{i=1}^{m} D_{(i)}-D_{\text {máx }}}{\sum_{i=1}^{m} D_{(i)}}\right]
$$

Em que:

$\mathrm{G}_{\mathrm{de}}=$ Grau de deterioração de um elemento estrutural;

$\mathrm{D}_{\text {máx }}=$ Maior grau de dano no elemento;

m: Número de danos detectados no elemento; e

$\mathrm{D}_{(\mathrm{i})}$ : O grau de dano de ordem (i).

Com base no valor encontrado para o $\mathrm{G}_{\mathrm{de}}$, tem-se a classificação do nível de deterioração e as medidas a serem tomadas, conforme Tabela 2.

Tabela 2 - Classificação dos níveis de deterioração do elemento $\left(\mathrm{G}_{\mathrm{de}}\right)$.

\begin{tabular}{c|c|c}
\hline $\begin{array}{c}\text { Nível de deterioração } \\
\text { Baixo }\end{array}$ & $\mathrm{G}_{\mathrm{de}}$ & Ações a serem tomadas \\
\hline Médio & $15-50$ & $\begin{array}{c}\text { Definir prazo/natureza para nova inspeção. Em } \\
\text { médio prazo (máx. 2 anos) }\end{array}$ \\
\hline Alto & $50-80$ & $\begin{array}{c}\text { Definir prazo para inspeção especializada detalhada. } \\
\text { Em curto prazo (máx. 1 ano) }\end{array}$ \\
\hline Sofrível & $80-100$ & $\begin{array}{c}\text { Definir prazo para inspeção especializada detalhada. } \\
\text { Em curto prazo (máx. 6 meses). }\end{array}$ \\
\hline Crítico & $>100$ & \begin{tabular}{c} 
Planejar intervenção imediata \\
\hline
\end{tabular}
\end{tabular}

Fonte: Adaptada de FONSECA (2007).

\subsection{Grau de Deterioração de uma família $\left(\mathbf{G}_{\mathrm{df}}\right)$}

Utiliza-se a Equação 03 apenas para elementos com nível de deterioração superior ou médio $\left(\mathrm{G}_{\mathrm{de}} \geq 15\right)$. Em caso de ser menor que 15 a Estrutura está em estado aceitável, realizar apenas a manutenção preventiva.

$$
G_{d f}=G_{d e m a ́ x} \sqrt{1+\frac{\sum_{i=1}^{m} G_{d e(i)}-G_{d e m a ́ x}}{\sum_{i=1}^{m} G_{d e(i)}}}
$$

Em que:

$\mathrm{G}_{\mathrm{df}}$ : Grau de deterioração de uma família de elementos;

$\mathrm{G}_{\text {demáx: }}$ Grau de deterioração máxima do elemento;

$\mathrm{G}_{\text {de(i): }}$ Grau de deterioração do elemento de ordem (i);

\subsection{Fator de Relevância Estrutural $\left(F_{r}\right)$}


O Fator de relevância estrutural tem por objetivo, considerar a importância relativa das diversas famílias que subdividem a estrutura, quanto ao comportamento e desempenho. Na tabela 3 tem-se a classificação do $\mathrm{F}_{\mathrm{r}}$.

Tabela 3 - Classificação do Fator de relevância estrutural $\left(\mathrm{F}_{\mathrm{r}}\right)$.

\begin{tabular}{c|l}
\hline $\mathrm{F}_{\mathrm{r}}$ & \multicolumn{1}{c}{ Elemento analisado } \\
\hline 1,0 & Elementos de composição arquitetônica \\
\hline 2,0 & Reservatório superior. \\
\hline 3,0 & $\begin{array}{l}\text { Escadas/rampas, reservatório inferior, cortinas, lajes } \\
\text { secundárias, juntas de dilatação. }\end{array}$ \\
\hline 4,0 & Lajes, fundações, vigas secundárias. \\
\hline 5,0 & Vigas e pilares principais. \\
\hline \multicolumn{2}{|c}{ Fonte: FONSECA (2007). }
\end{tabular}

\subsection{Grau de Deterioração da Estrutura $\left(G_{d}\right)$}

Conforme as informações obtidas anteriormente é possível calcular o grau de deterioração da Estrutura (Equação 04) e classificá-lo de acordo com a Tabela 4.

$$
G_{d}=\frac{\sum_{i=l}^{K} F_{r(i)} G_{d f(i)}}{\sum F_{r(i)}}
$$

Tabela 4- Classificação dos níveis de deterioração da estrutura $\left(\mathrm{G}_{\mathrm{d}}\right)$.

\begin{tabular}{|c|c|c|}
\hline Nível de deterioração & $\mathrm{G}_{\mathrm{d}}$ & Ações a serem tomadas \\
\hline Baixo & $0-15$ & Estado aceitável. Manutenção preventiva. \\
\hline Médio & $15-50$ & $\begin{array}{c}\text { Definir prazo/natureza para nova inspeção. Em médio prazo (máx. } 2 \\
\text { anos) }\end{array}$ \\
\hline Alto & $50-80$ & $\begin{array}{l}\text { Definir prazo para inspeção especializada detalhada. Em curto prazo } \\
\qquad \text { (máx. } 1 \text { ano) }\end{array}$ \\
\hline Sofrível & $80-100$ & $\begin{array}{l}\text { Definir prazo para inspeção especializada detalhada. Em curto prazo } \\
\text { (máx. } 6 \text { meses). }\end{array}$ \\
\hline Crítico & $>100$ & Planejar intervenção imediata \\
\hline
\end{tabular}

\section{ESTUDO DE CASO}

A partir da consolidação do fundamento teórico, realizou-se a inspeção predial no Prédio Administrativo do Centro Federal de Educação Tecnológica de Minas Gerais - CEFET/MG - Campus Curvelo, instituição que oferece o curso de Graduação em Engenharia Civil além dos cursos de nível médio técnico em Edificações, Eletrotécnica e Meio Ambiente. A edificação em questão é utilizada todos os dias para fins acadêmicos, tendo grande circulação de pessoas. Sendo assim, deve ser capaz de atender às necessidades de seus usuários com segurança.

A construção do edificio foi finalizada no ano de 2011, e esse está localizado na Rua Raymundo Mattoso, $\mathrm{n}^{\circ}$ 900, bairro Santa Rita, no Município de Curvelo/MG, coordenadas geográficas $18^{\circ} 44^{\prime} 37.86^{\prime \prime} \mathrm{S}$ e $44^{\circ} 26^{\prime} 46.72^{\prime \prime} \mathrm{O}$ (Figura 2). Trata-se de uma edificação em concreto armado, possibilitando a aplicação da Metodologia GDE/UnB, por meio de inspeções visuais. 


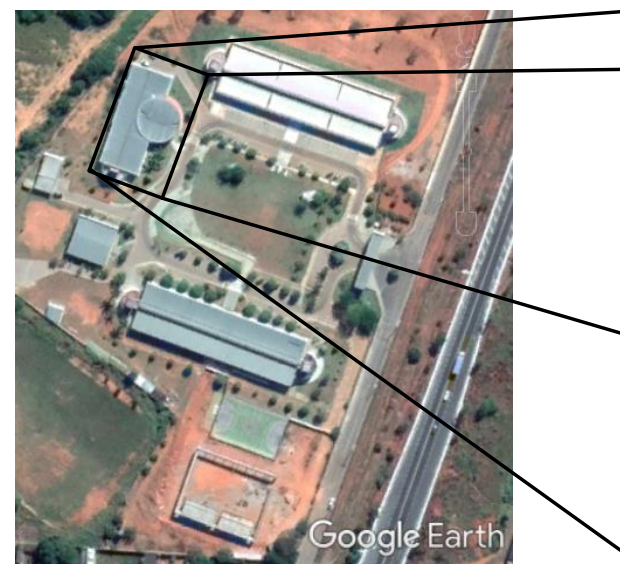

(a)

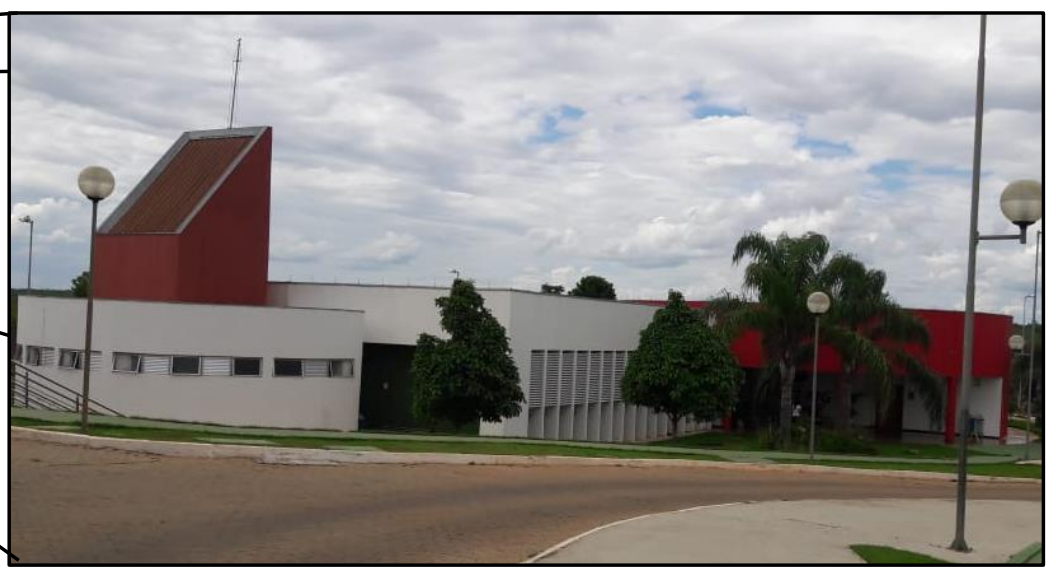

(b)

Figura 2- Localização da edificação em estudo. (a) Imagem de satélite. (b) Vista frontal. Fonte: esquerda - Google Earth (2019) e direita - autores.

\section{METODOLOGIA}

Para o seu desenvolvimento, no primeiro momento, foram detalhadas através de pesquisas bibliográficas as características dessa metodologia e as principais causas das manifestações patológicas que podem ocorrer nesse tipo de estrutura. Em seguida, realizou-se a inspeção predial do Prédio Administrativo do CEFET/MG - Campus Curvelo, de forma a identificar as anomalias e as falhas estruturais. Posteriormente, fez-se a avaliação da condição estrutural desse edifício onde calculou-se o grau de deterioração da estrutura. Para essa determinação, seguiu-se as seguintes etapas:

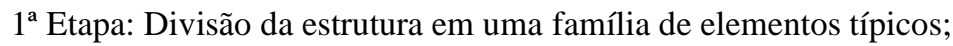

$2^{\text {a }}$ Etapa: Consulta do caderno de inspeção para cada elemento da família;

$3^{\mathrm{a}}$ Etapa: Atribuição do Fator de Ponderação do Dano $\left(\mathrm{F}_{\mathrm{p}}\right)$ e do Fator de Intensidade do Dano $\left(\mathrm{F}_{\mathrm{i}}\right)$;

$4^{\mathrm{a}}$ Etapa: Com os valores obtidos, é possível calcular o Grau do Dano (D);

$5^{\mathrm{a}}$ Etapa: Cálculo do Grau de deterioração do elemento $\left(\mathrm{G}_{\mathrm{de}}\right)$;

$6^{\mathrm{a}}$ Etapa: Cálculo do Grau de Deterioração da família de elementos $\left(\mathrm{G}_{\mathrm{df}}\right)$;

$7^{\mathrm{a}}$ Etapa: Introdução do fator de relevância estrutural da família $\left(\mathrm{F}_{\mathrm{i}}\right)$;

$8^{\mathrm{a}}$ Etapa: Cálculo do Grau de Deterioração da Estrutura $\left(\mathrm{G}_{\mathrm{d}}\right)$.

\section{RESULTADOS E DISCUSSÕES}

Para o cálculo de deterioração da estrutura, os elementos estruturais foram divididos nas seguintes famílias: elementos de composição arquitetônica, juntas de dilatação e vigas secundárias, uma vez que as demais famílias não apresentaram sinais de dano.

Durante a inspeção, foram identificadas as seguintes patologias: solapamento do solo e piso, fissuras na base de alguns elementos em alvenaria, fissuras agravadas por fluidos, solapamento do solo e piso com surgimento de vegetação, infiltrações em paredes, infiltração devido a falta de vedação externa, descascamento da pintura, descolamento do piso e fissuras na alvenaria.

As figuras 3 a 12 apresentam as patologias detectadas. 


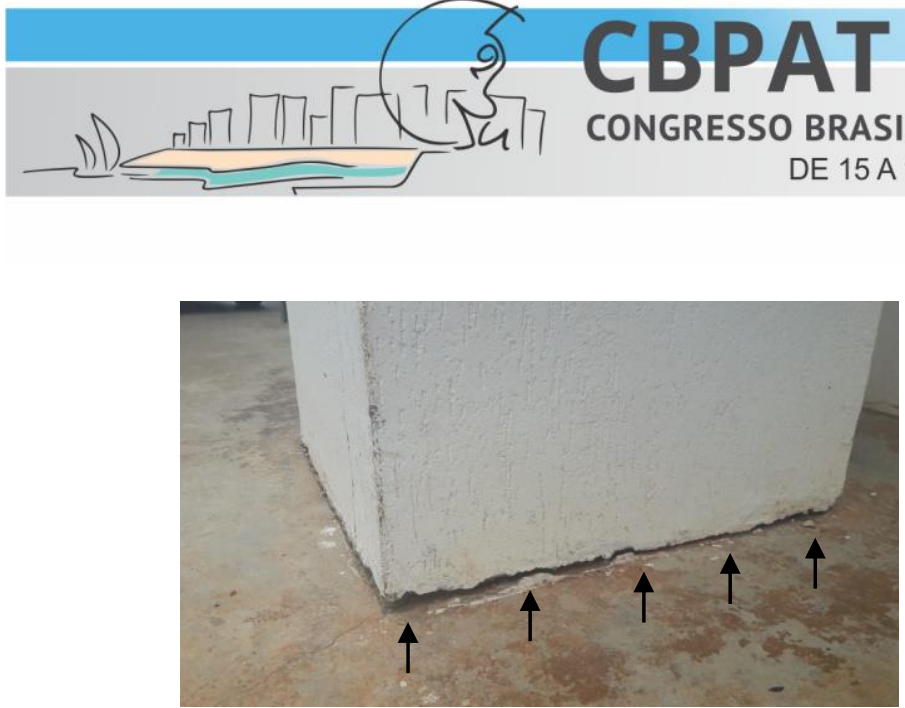

Figura 3 - Solapamento do solo e piso. Fonte: Autores.

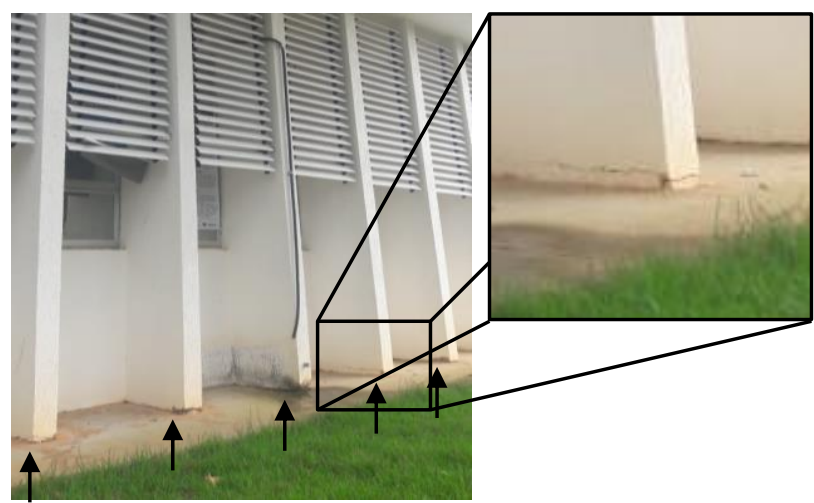

Figura 5 - Fissura na base dos elementos em alvenaria. Fonte: Autores.

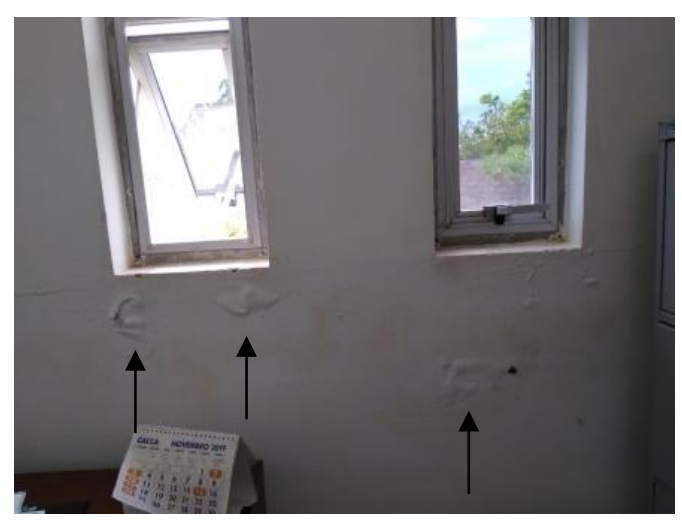

Figura 7 - Infiltração na parede. Fonte: Autores.

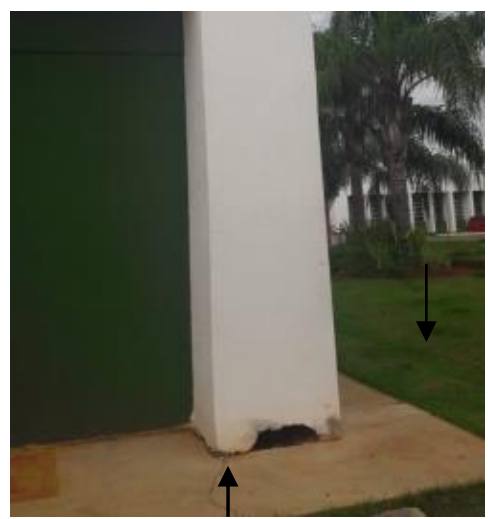

Figura 9 - Fissura (ausência de base de assentamento). Fonte: Autores.

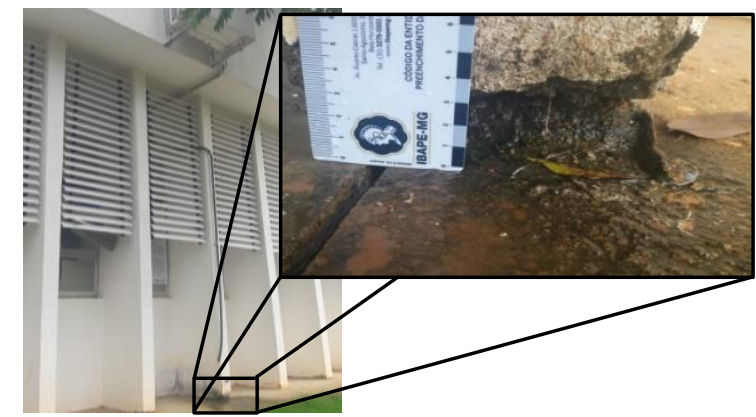

Figura 4 - Fissura agravada por umidade constante. Fonte: Autores.

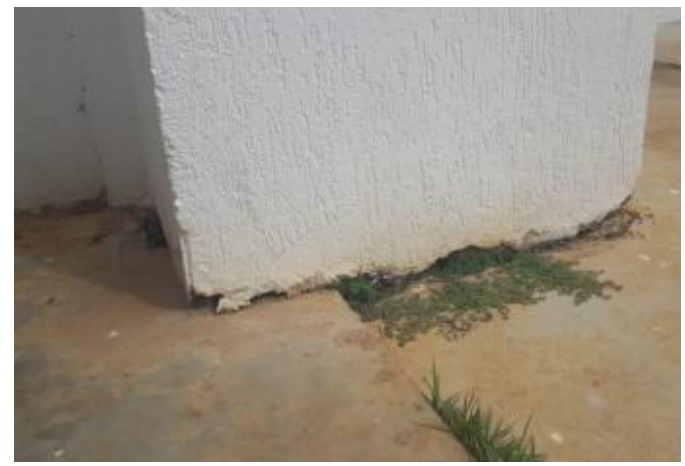

Figura 6 - Solapamento com surgimento de vegetação. Fonte: Autores.

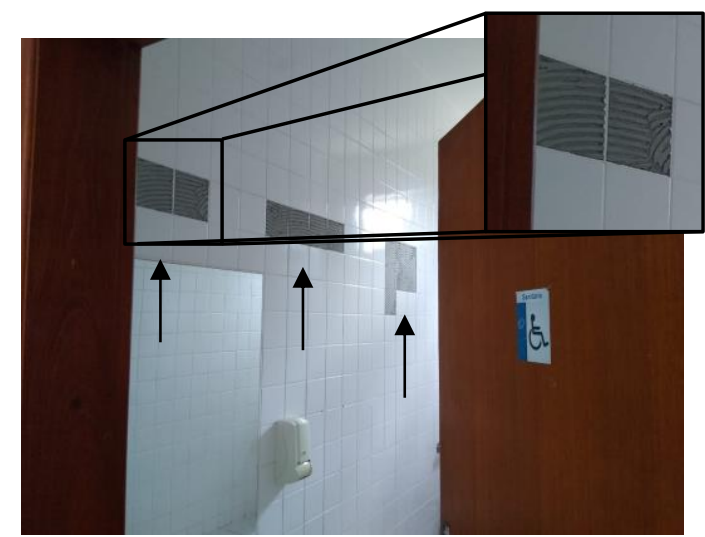

Figura 8 - Desplacamento do revestimento. Fonte: Autores.

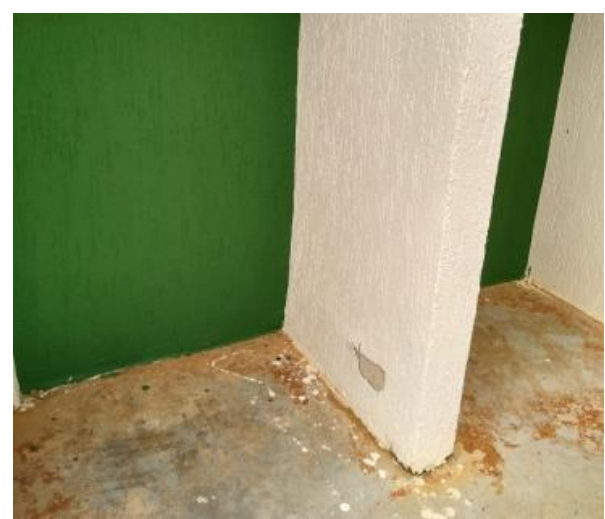

Figura 10 - Descascamento da pintura. Fonte: Autores. 


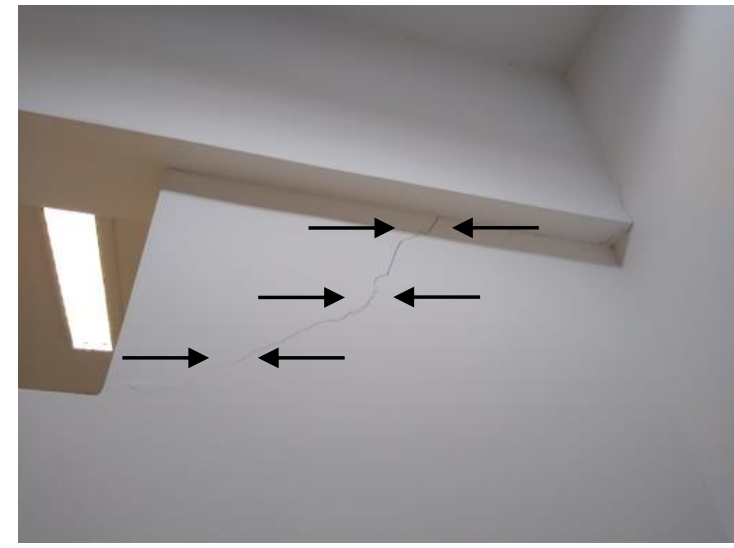

Figura 11 - Fissura na alvenaria. Fonte: Autores.

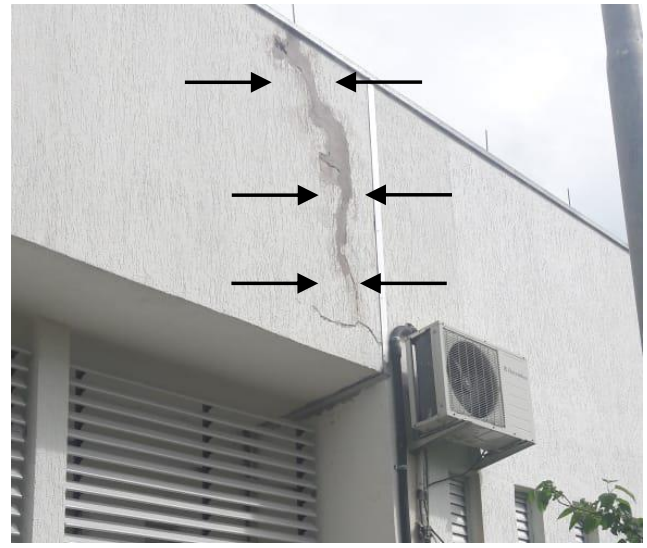

Figura 12 - Fissura próxima às juntas de dilatação. Fonte: Autores.

A partir dos fatores de intensidade $\left(\mathrm{F}_{\mathrm{i}}\right)$ e fatores de ponderação $\left(\mathrm{F}_{\mathrm{p}}\right)$ referentes a cada patologia e fator de relevância $\left(F_{\mathrm{r}}\right)$ de cada elemento foi possível o cálculo do grau de dano (D), grau de deterioração do elemento $\left(\mathrm{G}_{\mathrm{de}}\right)$, grau de deterioração da família de elementos tipos $\left(\mathrm{G}_{\mathrm{df}}\right)$ e grau de deterioração da estrutura $\left(\mathrm{G}_{\mathrm{d}}\right)$. Os resultados são apresentados na Tabela 5 .

Tabela 5- Cálculo do Grau de Deterioração da Estrutura $\left(\mathrm{G}_{\mathrm{d}}\right)$.

\begin{tabular}{|c|c|c|c|c|c|c|c|c|}
\hline Família & $\mathbf{F}_{\mathbf{r}}$ & Elemento & Patologia & $\mathbf{F}_{\mathbf{p}}$ & $\mathbf{F}_{\mathbf{i}}$ & D & $\mathbf{G}_{\mathbf{d e}}$ & $\mathbf{G}_{\text {df }}$ \\
\hline \multirow{9}{*}{$\begin{array}{l}\text { Elementos de } \\
\text { composição } \\
\text { arquitetônica }\end{array}$} & \multirow{9}{*}{1} & Figura 3 & Solapamento solo & 3 & 2 & 4,8 & \multirow{9}{*}{38,2} & \multirow{9}{*}{38,2} \\
\hline & & Figura 5 & $\begin{array}{c}\text { Fissura na base do } \\
\text { elemento }\end{array}$ & 3 & 2 & 4,8 & & \\
\hline & & Figura 4 & $\begin{array}{c}\text { Fissura agravada por } \\
\text { umidade constante }\end{array}$ & 3 & 2 & 4,8 & & \\
\hline & & Figura 6 & $\begin{array}{c}\text { Solapamento com } \\
\text { surgimento de vegetação }\end{array}$ & 3 & 2 & 4,8 & & \\
\hline & & Figura 7 & Infiltração na parede & 3 & 2 & 4,8 & & \\
\hline & & Figura 9 & $\begin{array}{l}\text { Fissura devido à ausência } \\
\text { de base de assentamento }\end{array}$ & 3 & 3 & 24 & & \\
\hline & & Figura 10 & Descascamento da pintura & 3 & 1 & 1,2 & & \\
\hline & & Figura 8 & $\begin{array}{c}\begin{array}{c}\text { Desplacamento do } \\
\text { revestimento }\end{array} \\
\end{array}$ & 3 & 2 & 4,8 & & \\
\hline & & Figura 11 & Fissura na alvenaria & 2 & 2 & 4,8 & & \\
\hline $\begin{array}{l}\text { Juntas de } \\
\text { dilatação }\end{array}$ & 3 & Figura 12 & $\begin{array}{l}\text { Fissura próximo às juntas } \\
\text { de dilatação }\end{array}$ & 3 & 3 & 12 & 12 & - \\
\hline \multicolumn{8}{|c|}{ GRAU DE DETERIORAÇÃO DA ESTRUTURA $\left(\mathbf{G}_{\mathbf{d}}\right)$} & 38,2 \\
\hline
\end{tabular}

Fonte: Autores

A partir dos valores obtidos, foi possível encontrar o grau de deterioração dos elementos de composição arquitetônica (Gd) igual a 38,2, indicando que deve-se definir prazo/natureza para nova inspeção em médio prazo (máx. 2 anos).

No caso das juntas de dilatação, tem-se o grau de deterioração desta família igual a 12, o que apresenta nível de deterioração baixo, sendo classificado como aceitável. Para este caso é necessária a realização de manutenção preventiva.

Com base nos valores encontrados, deve-se calcular o Grau de Deterioração da família $\left(\mathrm{G}_{\mathrm{df}}\right)$ e Grau de Deterioração do da estrutura $\left(\mathrm{G}_{\mathrm{d}}\right)$ apenas a partir dos Elementos de Composição Arquitetônica, visto que a outras famílias avaliadas foi obtido $\mathrm{G}_{\mathrm{df}}$ inferior a 15. As demais famílias do edifício em questão não apresentaram sinais de danos. Sendo assim, 
tem-se o Grau de deterioração da estrutura igual a 38,2. Para esse valor de deterioração da Estrutura tem-se que é preciso prazo/natureza para nova inspeção. Em médio prazo (máx. 2 anos).

\section{CONCLUSÕES}

A partir deste trabalho, verifica-se que a Metodologia GDE/UnB identificou uma quantidade considerável de danos na família de elementos de composição arquitetônica, sendo necessário realizar uma intervenção em médio prazo. A eficiência da metodologia é evidenciada ao analisar os elementos de forma isolada, possibilitando intervenções na família desejada. Esta é uma ferramenta que auxilia no cadastramento das patologias, assim como no acompanhamento de suas evoluções, além de ser importante para um processo futuro de reabilitação.

Além disso, é importante salientar que esta Metodologia não avalia a condição das instalações elétricas e hidráulicas do edificio. Sendo assim, é importante sugerir que as mesmas sejam avaliadas em trabalhados futuros, principalmente por se tratar de um edificio escolar frequentado diariamente por estudantes, professores e colaboradores.

\section{AGRADECIMENTOS}

Agradecemos ao Centro Federal de Educação Tecnológica de Minas Gerais - CEFET-MG pelo apoio direto e indireto a este trabalho, em especial ao LAR - Laboratório de Avaliação e Reabilitação de Ambiente Construído do CEFET-MG Campus Curvelo. 


\section{REFERÊNCIAS}

ASSOCIAÇÃO BRASILEIRA DE NORMAS TÉCNICAS (ABNT). NBR 5674: Manutenção de edificações Requisitos para o sistema de gestão de manutenção. Rio de Janeiro: ABNT, 2012.

BOAS, E. et al . Aplicação de Metodologia GDE/UnB em uma edificação habitacional: Estudo de caso em Águas Lindas de Goiás- GO. CONGRESSO BRASILEIRO DO CONCRETO, Goías, 2018. Disponível em $<$ http://ibracon.org.br/eventos/adminx/artigos/60CBC2024-ver4-autor-

APLICA\%C3\%87\%C3\%830\%20DE\%20METODOLOGIA\%20GDE\%20UnB\%20EM\%20UMA\%20EDIFICA\%C3\% 87\%C3\%83O $\% 20$ HABITACIONAL $\% 20 \% 20$ ESTUDO $\% 20$ DE $\% 20$ CASO $\% 20$ EM $\% 20 \%$ C3\%81GUAS\%20LINDAS \%20DE\%20GOI\%C3\%81S-\%20GO.pdf> . Acesso em: 07 set. 2019.

BOLDO, P., Análise Quantitativa de Estruturas de Concreto Armado de Edificações no Âmbito do Exército Brasileiro. Dissertação (Mestrado em Estruturas), Departamento de Engenharia Civil e Ambiental, Universidade de Brasília, Brasília, DF, 2002.

CASTRO, E. K., Desenvolvimento de Metodologia para Manutenção de Estruturas de Concreto Armado. Dissertação (Mestrado em Estruturas), Departamento de Engenharia Civil, Universidade de Brasília, Brasília, DF, 1994.

FONSECA, R.P., A estrutura do Instituto Central de Ciências: Aspectos históricos, científicos e tecnológicos de projeto, execução, intervenções e propostas de manutenção. Dissertação (Mestrado em Estruturas e Construção Civil), Departamento de Engenharia Civil e Ambiental, Universidade de Brasília. Brasília, DF, 2007.

GOOGLE. Google Earth. Disponível em <http://earth.google.com/>. Acesso em: 08 jan 2020.

HELENE, P. Manutenção para Reparo, Reforço e Proteção de Estruturas de Concreto. $2^{\mathrm{a}}$ ed. SP: Pini, 1992.

Instituto Brasileiro de Avaliações e Perícias de Engenharia (IBAPE). Norma de Inspeção Predial, 2012. Disponível < http://ibape-nacional.com.br/biblioteca/wp-content/uploads/2012/12/Norma-de-Inspe\%C3\%A7\%C3\%A3o-Predial-

IBAPE-Nacional.pdf > Acesso em: 23 ago. 2019.

LOPES, B.A.R., Sistema de manutenção estrutural para grandes estoques de edificações: Estudo para a inclusão do componente Estrutura de Concreto. Dissertação (Mestrado em Estruturas), Departamento de Engenharia Civil, Universidade de Brasília, Brasília, DF,1998. 